\title{
Advanced testicular cancer associated with life-threatening tumour lysis syndrome and choriocarcinoma syndrome
}

\author{
Kohei Kobatake, MD; Masao Kato, MD; Koji Mita, MD \\ Department of Urology, Hiroshima City Asa Hospital, Hiroshima, Japan
}

Cite as: Can Urol Assoc J 2015;9(1-2):62-4. http://dx.doi.org/10.5489/cuaj.2499

Published online February 5, 2015.

\section{Abstract}

Tumour lysis syndrome (TLS) and choriocarcinoma syndrome (CS) are severe complications of chemotherapy for testicular cancer. They are rare, but can be life-threatening. A 37-year-old man complaining of persisting cough was referred to our hospital. A computed tomography $(\mathrm{CT})$ scan revealed huge tumours that occupied the peritoneal cavity, with multiple lung, liver, and para-aortic metastases. Although there was no abnormal finding in the testes, serum testicular tumor markers showed marked elevation. A CT-guided biopsy for the peritoneal tumours revealed extragonadal germ cell tumour (GCT), including yolk sac tumour and choriocarcinoma. Chemotherapy with bleomycin, etoposide, and cisplatin (BEP) was started after admission. The morning after the beginning of BEP, the patient developed hemorrhagic shock, in addition to acute pulmonary and renal failure, because of TLS and massive hemorrhage at bilateral lung metastases. He was intubated and resuscitated. Despite appropriate therapy, his renal function did not recover and hemodialysis was started. The patient eventually died of severe respiratory distress syndrome and infection. To our knowledge, this is the first case report of TLS and CS as complications of hemorrhage at the lung metastases of advanced testicular cancer leading to death.

\section{Introduction}

One of the standard chemotherapies for advanced testicular cancer is BEP (bleomycin, etoposide, and cisplatin). This chemotherapy has serious adverse effects, such as choriocarcinoma syndrome (CS) and tumor lysis syndrome (TLS). CLS can manifest as hemorrhage at metastatic sites and can cause acute pulmonary disorder when those sites are in the lungs; ${ }^{1,2}$ TLS can be caused by antineoplastic-drug-sensitive tumours or bulky tumours.

We present the clinical course of an extragonadal germ cell tumour cancer in a patient who died after developing both CS and TLS. To our knowledge, this is the first report of
TLS and CS as complications of the hemorrhage at the lung metastases in advanced testicular cancer leading to death.

\section{Case report}

A 37-year-old man complaining of persisting cough was referred to our hospital. He had multiple shadows in his chest x-rays. A computed tomography (CT) scan revealed multiple bilateral nodules, including up to $50-\mathrm{mm}$ lesions in both lungs and huge tumours (up to $188 \mathrm{~mm}$ in diameter) in the peritoneal cavity (Fig. 1), multiple liver nodules up to $44 \mathrm{~mm}$ in diameter, and multiple para-aortic lymph node enlargements (Fig. 2a). Furthermore, there was bilateral hydronephrosis due to the abdominal tumour.

While there was no abnormal finding of the testes, marked elevation of serum testicular tumour markers was noted with human chorionic gonadotropin (hCG) $150670 \mathrm{mIU} / \mathrm{mL}$, $\beta$-hCG $9772 \mathrm{ng} / \mathrm{mL}, \alpha$-fetoprotein (AFP) $2232 \mathrm{ng} / \mathrm{dL}$, and lactate dehydrase (LDH) $1682 \mathrm{IU} / \mathrm{L}$. The pathology of the CT-guided biopsy for the peritoneal tumors revealed an extragonadal germ cell tumour (GCT), including yolk sac tumor and choriocarcinoma. We started chemotherapy with BEP (single-dose bleomycin [30 mg]), etoposide [100 mg/ $\left.\mathrm{m}^{2}\right]$, and cisplatin $\left[20 \mathrm{mg} / \mathrm{m}^{2}\right]$ ) on day 1 after placing bilateral ureteral stents to preserve renal function. In the morning on day 2 , the patient showed oliguria, hypotension, and drastic dyspnea. He was transferred to the intensive care unit, leading to intubation and mechanical ventilation because of rapidly developing acute respiratory insufficiency. A CT scan revealed severe bilateral lung metastases (Fig. 2b), in addition, the serum hemoglobin level decreased to $5.3 \mathrm{~g} / \mathrm{dL}$ and the serum creatinine level increased to $4.74 \mathrm{mg} / \mathrm{dL}$. Hyperkalemia and hypocalcemia also appeared.

Blood tests and the patient's vital signs showed that TLS and a hemorrhagic shock due to intrathoracic hemorrhage from pulmonary metastases occurred simultaneously. Immediately after bilateral thoracentesis to remove the compression by the hemothorax, $900 \mathrm{~mL}$ of blood was evacuat- 


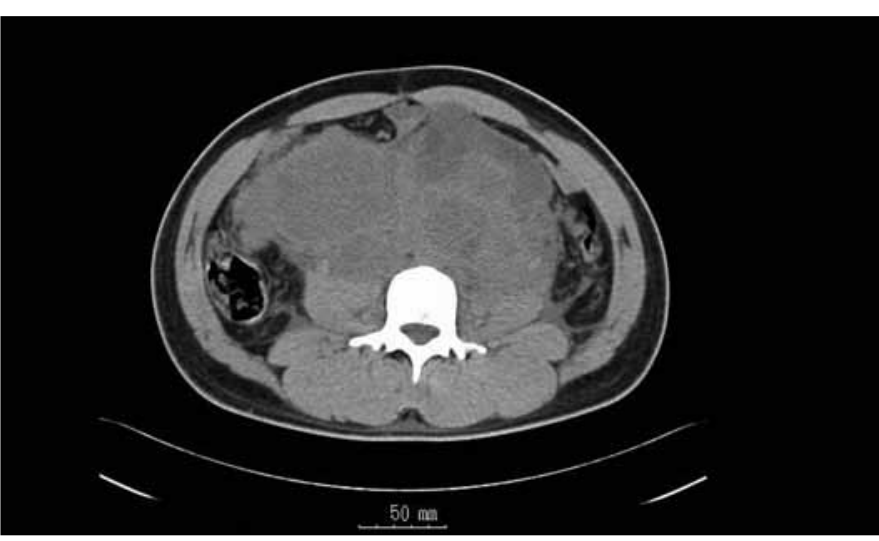

Fig. 1. Primary extragonadal germ cell tumours.

ed. Operative techniques to stop the bleeding were thought to be difficult because the respiratory specialist we consulted thought that there might be multiple bleeding sites in both lungs. We therefore managed the bleeding conservatively using styptics. The hemorrhage was marginally controlled, and the thoracostomy tube was removed on day 7 because of the decrease of drainage.

The acute renal failure was suspected to be due to both hypovolemia and TLS, so blood, sodium bicarbonate, and allopurinol were infused intravenously. The renal failure, however, kept exacerbating, so hemodialysis was started on day 4.

Meanwhile, bone marrow suppression was evident on day 14, and septic shock and disseminated intravascular coagulation were evident on day 18. Despite administration of several antibiotics and $\gamma$ globulin, on day 21 the patient died of the exacerbation of respiratory failure due to the severe respiratory infection and respiratory distress syndrome.

\section{Discussion}

We present the clinical course of an advanced metastatic extragonadal GCT case with life-threatening pulmonary hemorrhage and TLS. Logothetis has described the hemorrhage at the site of metastasis in advanced GCTs with high volume choriocarcinomatous elements and termed it "choriocarcinoma syndrome ${ }^{\prime 1}$ or CS.

Both TLS and CS are rare, but sometimes lethal. While there are several case reports of either TLS or CS in which the patient was able to be saved, there is only 1 in which both the syndromes occurred at the same time (liver and small intestine hemorrhages associated with hyperuricemia). ${ }^{3}$

$\mathrm{CS}$ is liable to occur in cases with significantly high hCG; tumour invasion into pulmonary small vessels can cause the syndrome. ${ }^{4}$ The mechanism and the frequency of occurrence is still unclear. Acute pulmonary hemorrhage is the most frequent manifestation of CS, but hemorrhages due to CS can develop at any site of metastasis: liver, brain, and small

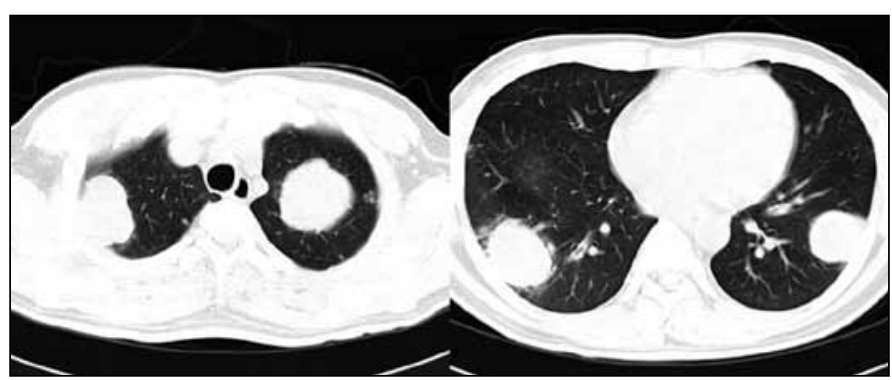

Fig. 2a. Bilateral lung metastases. Chest computed tomography before initiation of bleomycin, etoposide, and cisplatin (BEP) showed multiple nodules.

bowel. ${ }^{3}$ It may develop immediately after chemotherapy, or in patients with rapidly progressing disease. ${ }^{2}$ There are some reports describing cases in which pulmonary bleeding was controlled by lobectomy. ${ }^{5}$

TLS is known as a syndrome developing commonly in hemodyscrasia, such as leukemia or malignant lymphoma. Physiolysis of the cancer cells or destruction of cancer tissue by chemotherapy makes the cancer cells release nucleic acid, potassium, phosphorus, cytokine, and uric acid as metabolites of nucleic acid. Furthermore, the release of cytokines can cause a systemic inflammatory response that sometimes leads to multiple organ failure. ${ }^{6}$

After Blonke reported the first case of TLS in $2000,{ }^{7}$ there have been similar reports about TLS in testicular cancer. These reports included a case associated with acute respiratory failure, but none with choriocarcinoma.

Known risk factors for TLS include an LDH level more than the twice the normal upper limit, intravital total tumour volume more than $10 \mathrm{~cm}$, and solid cancer with a proliferating ability and higher sensitivity to treatment. ${ }^{8}$ Adequate hydration and the administration of allopurinol and rasburicase are recommended, but these preventive measures might be inadequate; one case describes acute renal failure occurring despite their use. ${ }^{9}$ Currently, there is also no effective prevention for CS.

\section{Conclusion}

These 2 syndromes are extremely rare and their onset is difficult to predict accurately, but they may lead to treatment-

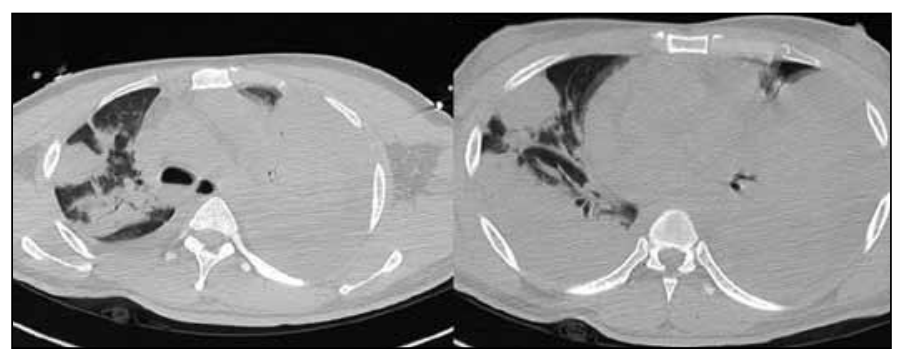

Fig. 2b. Bilateral lung metastases. Chest computed tomography on day 2 of bleomycin, etoposide, and cisplatin (BEP), immediately after onset of choriocarcinoma syndrome. 
Kobatake et al.

related death. The risk of these complications should be recognized; in addition, physicians should explain the risk to patients starting chemotherapy for a testicular cancer with significantly high hCG, a huge primary lesion, or metastatic lesions.

Competing interests: Authors declare no competing financial or personal interests.

This paper has been peer-reviewed.

\section{References}

1. Logothetis CJ. Choriocarcinoma syndrome. Cancer Bull 1984;36:118-20.

2. Motzer RJ, Bosl GJ. Hemorrhage: A complication of metastatic testicular choriocarcinoma. Urology 1987;30:119-22. http://dx.doi.org/10.1016/0090-4295(87)90175-0
3. Kawai K, Takaoka E, Naoi M, et al. A case of metastatic testicular cancer complicated by tumour lysis syndrome and choriocarcinoma syndrome. Jpn J Clin Oncol 2006;36:665-7. http://dx.doi.org/10.1093/ iico/hyl078

4. Shintaku M, Hwang MH, Amitani R. Primary choriocarcinoma of the lung manifesting as diffuse alveolar hemorrhage. Arch Pathol Lab Med 2006;130:540-3.

5. Tatokoro M, Kawakami S, Sakura M, et al. Successful management of life-threatening choriocarcinomo syndrome with rupture of pulmonary metastatic foci causing hemorrhagic shock. Int J Urol 2008; 15:263-4. http://dx.doi.org/10.1111/j.1442-2042.2007.01979.x

6. Hiijiya N, Metzger ML, Pounds S, et al. Severe cardiopulmonary complication consistent with systemic inflammatory response syndrome caused by leukemia cell lysis in childhood acute myelomyocytic or monocytic leukemia. Pediatr Blood Cancer 2005;44:63-9. http://dx.doi.org/10.1002/pbc.20192

7. Blanke CD. Acute tumor lysis syndrome with choriocarcinoma. South Med J 2000;93:916-9. http:// dx.doi.org/10.1097/00007611-200093090-00017

8. Coiffire $\mathrm{B}$, Altman A, Pui $\mathrm{CH}$, et al. Guidelines for the management of pediatric and adult tumor lysis syndrome: An evidence-based review. J Clin Oncol 2008;26:2767-78. http://dx.doi.org/10.1200/ JC0.2007.15.0177

9. Feres $\mathrm{GA}$, Salluh Il, Ferreira $\mathrm{CG}$, et al. Severe acute tumor lysis syndrome in patients with germ-cell tumors. Indian J Urol 2008;24:555-7. http://dx.doi.org/10.4103/0970-1591.44267

Correspondence: Dr. Kohei Kobatake, Department of Urology, Hiroshima City Asa Hopital, 2-1-1, Kabe-minami, Asakita-ku, Hiroshima 731-0293, Japan; koukoba2710@gmail.com 